\title{
Noble Gas Fractionation: Some insights from molecular simulations
}

GUILlaUme GALliero $^{1 *}$, HAI HOANG ${ }^{2}$, MAGALI PUJOL $^{3}$

${ }^{1}$ LFCR, Universite de Pau et des Pays de l'Adour, E2S UPPA, CNRS, TOTAL, UMR 5150, 64000 Pau, France

(*correspondence : guillaume.galliero@univ-pau.fr)

${ }^{2}$ IFAS, Duy Tan University, 10C Tran Nhat Duat Street, District 1, Ho Chi Minh City 70000, Viet Nam.

${ }^{3}$ TOTAL EP, CSTJF, Avenue Larribau, 64000 Pau, France.

Noble gases are widely used as natural tracers to characterize storage, migration and origin of fluids in geological environments. Interestingly, they are only fractionated by physical phenomena, such as phase partitioning, mass diffusion or thermodiffusion. However, the quantification, and the modelling, of the related physical parameters (Henry's constant, diffusion coefficient, thermal diffusion factor) is not an easy task especially when dealing with petroleum systems [1].

To address the quantification and the modelling of noble gas fractionation in natural energy resources, molecular simulation is an appealing option as this numerical tool provides complementary information to experiments. Thus, in this work, we have used molecular simulations [2] to provide quasi experimental data on noble gas phase partitioning, mass diffusion and thermodiffusion in model petroleum fluids and in water, both in bulk fluids and in nanoporous medium, helping to revisit the existing models.

Among others, it has been found that: (i) Noble gas partitioning between gas and oil depends not only on density but on oil composition as well [3]; (ii) the "square root relationship" is inadequate to describe isotopic fractionation by mass diffusion [4]; (iii) a typical geothermal gradient has an impact on vertical noble gas isotopic and elemental fractionation which is of the same order of magnitude than gravity segregation [5]; (iv) both solubility and mass diffusion of noble gas in water are strongly affected by clay confinement [6].

[1] Ballentine et al. (2002), Rev. Min. Geoch. 47539. [2] Hoang et al. (2017), Ind. Chem. Eng. Res. 56, 9213. [3] Hoang et al., (in preparation). [4] Seltzer et al. (2019) EPSL 514, 156. [5] Hoang et al. (2019) Eur. Phys. J. E. 42, 61. [6] Gadikota et al. (2017), J. Phys. Chem. C, 121, 26539. 